\title{
Machine learning surrogate models for prediction of point defect vibrational entropy
}

\author{
Clovis Lapointe, ${ }^{1, *}$ Thomas D. Swinburne,${ }^{2, \dagger}$ Louis Thiry, ${ }^{3}$ Stéphane Mallat, ${ }^{4}$ \\ Laurent Proville, ${ }^{1}$ Charlotte S. Becquart, ${ }^{5}$ and Mihai-Cosmin Marinica ${ }^{1, \ddagger}$ \\ ${ }^{1}$ DEN-Service de Recherches de Métallurgie Physique, CEA Saclay, 91191 Gif-sur-Yvette, France \\ ${ }^{2}$ CINaM, CNRS Aix-Marseille, 13009 Marseille, France \\ ${ }^{3}$ Département d'informatique, ENS, CNRS, PSL University, Paris, France \\ ${ }^{4}$ Collège de France, ENS, PSL University, Paris, France. Flatiron Institute, New-York, USA. \\ ${ }^{5}$ Univ. Lille, CNRS, INRA, ENSCL, UMR 8207 - UMET - Unité Matériaux et Transformations, F-59000 Lille, France
}

(Dated: June 22, 2020)

\begin{abstract}
The temperature variation of the defect densities in a crystal depends on vibrational entropy. This contribution to the system thermodynamics remains computationally challenging as it requires a diagonalisation of the system's Hessian which scales as $O\left(N^{3}\right)$ for a crystal made of $\mathrm{N}$ atoms. Here, to circumvent such an heavy computational task and make it feasible even for systems containing millions of atoms the harmonic vibrational entropy of point defects is estimated directly from the relaxed atomic positions through a linear-in-descriptor machine learning approach of order $O(N)$. With a size-independent descriptor dimension and fixed model parameters, an excellent predictive power is demonstrated on a wide range of defect configurations, supercell sizes and external deformations well outside of the training database. In particular, formation entropies in a range of 250 $k_{B}$ are predicted with less than $1.6 k_{B}$ error from a training database whose formation entropies span only $25 k_{B}$ (train error less than $1.0 k_{B}$ ). This exceptional transferability is found to hold even when the training is limited to a low energy superbasin in the phase space while the tests are performed for a different liquid-like superbasin at higher energies.
\end{abstract}

Keywords: Vibrational Entropy, Defects, Machine Learning, Molecular Dynamics, Harmonic approximation, Empirical Potentials

\section{INTRODUCTION}

The aging of crystalline materials is heavily influenced by the thermodynamic and kinetic properties of point defects. Their evolution gives rise to an extraordinarily diverse range of defect morphologies [1-8] whose distributions in size, character and density exhibit significant variations with temperature.

The stability of defect populations changes in response to temperature variation [9-12] according to the system entropy in which one distinguishes three distinct contributions associated to : (i) various geometry configurations [13], (ii) electronic thermal excitations [14] and (iii) lattice thermal vibrations [15]. For an isolated vacancy close to melting temperature, both electronic and vibrational entropies have same order of magnitude [14] around $3 k_{B} / 2$ while configurational entropy reduces to the mixing entropy and thus is negligible in dilute systems [15]. Below the melting temperature, the electronic entropy decreases linearly in temperature as the width of the Fermi surface sharpens. The vibrational contribution becomes thus dominant up to few kelvins where quantum effects yields an abrupt decrease, similar to the phonon heat capacity. For more complex defects, the configurational entropy is augmented by a term $k_{B} \ln \left(\mathcal{N}_{c}\right)$ where $\mathcal{N}_{c}$ is the number of different geometries corresponding to

\footnotetext{
* clovis.lapointe@cea.fr

$\dagger$ swinburne@cinam.univ-mrs.fr

‡ mihai-cosmin.marinica@cea.fr
}

the same internal energy. Since it does not vary with temperature, this contribution does not affect the stability of defect structures. We shall also leave aside the temperature dependence of the internal energy which is inherent to the thermal expansion of solids [15]. Our study is devoted to the computation of the vibrational entropy as it represents surely [16] an important contribution to the stability of defect in a wide range of temperature.

For a solid containing $N$ atoms, the standard harmonic approximation of entropy [17] requires a $\mathcal{O}\left(N^{2}\right)$ calculation of the dynamical matrix and a $\mathcal{O}\left(N^{3}\right)$ diagonalisation to find the vibrational spectrum. The procedure is schematically represented in Fig.1(a). For instance, the computational load for such a task in a crystal made of $2 \times 10^{5}$ atoms requires more than $20 \mathrm{~TB}$ of memory and ten hours over thousands of the most recent CPUs. Different methods have been developed [18-26] to compute directly the free energy of defects including the nonharmonic contributions from energy and entropy in an indistinguishable manner. However these methods remain computationally very heavy as they usually rely on sampling the system phase space through the construction of random or optimized trajectories. The essential problem arises from the convergence of such methods as to achieve a reliable sampling the number of iterations needed scales as $\mathcal{O}\left(N^{2}\right)$ or $\mathcal{O}\left(N^{3}\right)$, in the more favorable case. Furthermore we notice that according to neutron scattering experiments, the non-harmonic contributions to the formation of defect are not essential to the computation of vibrational entropy in a broad range of temperature, i.e. up to $700 \mathrm{~K}$ for example in $\alpha$-Fe [27] and Al [28]. 
In the present study we thus propose a surrogate model to evaluate the harmonic vibrational entropy using a linear-in-descriptor machine learning (LDML) approach with $\mathcal{O}(N)$ computational cost [29-38]. The method is applied to a wide class of point defects using only the relaxed atomic positions to determine directly the vibrational entropy. We chose to exemplify our computational technique using empirical potential interactions in $\alpha$-Fe. The accuracy of interatomic potentials is currently undergoing a renaissance due to ever larger databases and new potential formalisms employing machine learning techniques [30, 34-37, 39-44], statistical on-the-fly learning [45, 46] and mixed elastic-atomic models [3, 47] amongst others. The empirical interatomic potential models employed here were fitted on ab initio [48] or experimental data [49]. Their relative simplicity allowed us rapidly asses a wide range of defect structures and to explore a large dataset in large crystals inaccessible via standard $a b$ initio methods [50-52].

Our main finding is that the LDML approach we propose exhibits an exceptional degree of transferability, giving the ability to rapidly asses defect vibrational entropy at realistic temperatures in different systems of bodycentered cubic (bcc) Fe containing defects. The same machine learning parametrisation allows us to predict the formation entropies of different defects over a wide range of $250 k_{B}$ to within an root mean square error (RMSE) inferior to $2 k_{B}$, despite the rather narrow training set having a total formation entropy range of only $25 k_{B}$.

In section II we define the harmonic vibrational entropy and emphasis the equivalence between the local and the eigen descriptions for phonons in the harmonic approximation. In section III we describe the machine-learning approach for vibrational entropy, by introducing the LDML model and the descriptor functions. In section IV the dataset production and model training is detailed before being applied to predict the formation entropy of various defects in section $\mathrm{V}$. The excellent transferability found in initial applications to point defects is pushed further in section VD, where our model trained on defect structures is applied to predict the formation entropy of high defected structures generated by random displacements in bcc iron supercell. The success of the present approach opens many perspectives for high-throughput, multiscale materials science calculations which are discussed in section VI.

\section{VIBRATIONAL ENTROPY IN THE HARMONIC APPROXIMATION}

To evaluate vibrational properties under the harmonic approximation we consider the normal modes of a system with $N$ atoms, obtained from the spectrum of the force constant matrix $\phi \in \mathbb{R}^{3 N \times 3 N}$ through

$$
\left(\phi-M \omega_{\nu}^{2}\right)|\nu\rangle=|0\rangle .
$$
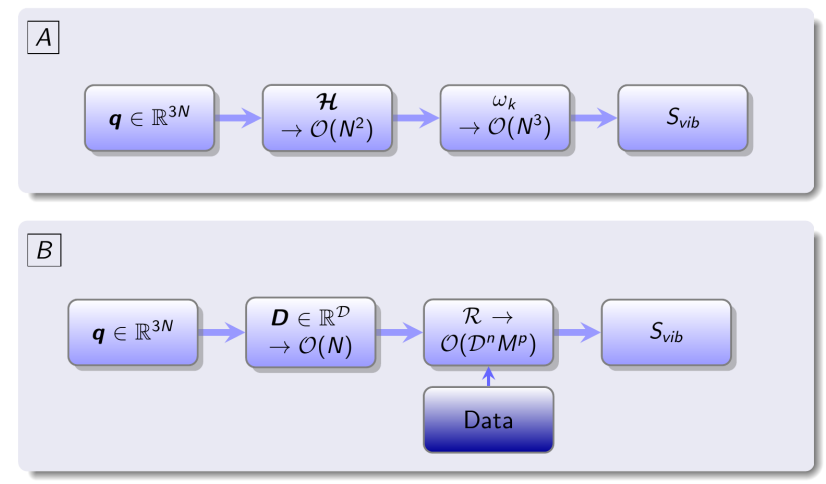

FIG. 1: Two strategies to evaluate the harmonic formation entropy of defects embedded into crystalline structure having $N$ atoms: (a) the traditional approach based on the phonons spectrum of Hessian $\mathcal{H}$ (the second derivatives of the potential energy of the system) (b) the machine learning surrogate model based on the $M$ instances of the database,

fitted via regression in the descriptor space $\mathbb{R}^{\mathcal{D}}$. The

descriptor or feature space is the representation of the atomic configuration through the descriptor functions. Both scaling coefficients of the regression $n$ and $p$ range between 0 and 2 depending on the method used. For the linear fit, used in the present study, $n=1$ and $p=0$.

Where $\boldsymbol{M} \in \mathbb{R}^{3 N \times 3 N}$ is a diagonal mass matrix. If we only consider phonons in the Debye approximation, appropriate for phonons near the center of the Brillouin zone, the force constant matrix becomes the Hessian operator $\mathcal{H}$, the matrix of second derivatives of the potential energy $U$. As discussed above, to obtain the eigenvalues $\omega_{\nu}^{2}$ and eigenvectors $|\nu\rangle$ the Hessian must be diagonalised. In the classical approximation, i.e. when the temperature is larger than the crystal Debye temperature such as $\frac{\hbar \omega_{\nu}}{k_{B} T} \ll 1 \forall|\nu\rangle$, the entropy becomes [53, 54]:

$$
S(T, N)=k_{B} \sum_{\nu}\left[\ln \left(\frac{k_{B} T}{\hbar \omega_{\nu}}\right)+1\right],
$$

where $k_{B}$ and $\hbar$ are the Boltzmann and Planck constants, respectively. For finite crystalline systems containing $N_{b}$ bulk atoms and $\pm N_{d}$ point defects, the vibrational formation entropy $S_{f}$ is defined as

$$
S_{f}\left(T, N_{d}\right)=S_{d}\left(T, N_{b} \pm N_{d}\right)-\frac{N_{b} \pm N_{d}}{N_{b}} S_{b}\left(T, N_{b}\right),
$$

where the entropies $S_{b}$ and $S_{d}$ of the bulk and defective systems are computed at the same volume $V$. With Hessian eigenvalues $\omega_{\nu_{b}}^{2}$ and $\omega_{\nu_{d}}^{2}$ for the bulk and defect systems, Eq.(2) yields a harmonic formation entropy :

$$
S_{f}\left(T, N_{d}\right)=k_{B} \ln \left(\frac{\prod_{\nu_{b}}\left(\hbar \omega_{\nu_{b}}\right)^{\frac{N_{b} \pm N_{d}}{N_{b}}}}{\prod_{\nu_{d}} \hbar \omega_{\nu_{d}}}\right) \text {. }
$$

Fig.1 schematically summarises the numerical treatment required to compute entropy through the diagonalisation of the Hessian matrix, which has an $\mathcal{O}\left(N^{3}\right)$ computational demand that typically prohibits application to 
large systems. We have to note that an $\mathcal{O}(N)$ approach has been developed by different authors [55], who treat the summation in Eq.(2) as an expectation value over the eigenvalue distribution. They approximated this distribution using a set of Chebyshev polynomials with a random basis set. Whilst this stochastic approach is indeed more efficient than $\mathcal{O}\left(N^{3}\right)$ treatment for large systems, a converged result requires a proper selection of a large set of polynomials and basis vectors, requiring a computational effort which is still impractically high for the high-throughput evaluation desired in the present work, motivating our use of LDML models.

\section{A. Green function formalism for vibrational entropy calculations}

Within the harmonic approximation, evaluation of the vibrational entropy requires knowledge of the full phonon spectrum, which can be directly computed from the secular equation Eq.(1). The Green function formalism is an alternative and elegant way to iteratively solve this same eigen-problem. Taking eigenmodes $|\nu\rangle$ the Greens function $\mathcal{G} \in \mathbb{C}^{3 \times 3} \otimes \mathbb{C}^{N \times N}$ writes [53]:

$$
\mathcal{G}(\omega)=\sum_{\nu} \frac{|\nu\rangle \otimes\langle\nu|}{\omega_{\nu}^{2}-\omega^{2}}
$$

The total phonon density of states is then the imaginary part of the trace of Green's function [53]

$$
\Omega(\omega)=\frac{2 \omega}{\pi} \Im(\operatorname{Tr}\{\mathcal{G}(\omega)\}),
$$

where $\Im(\cdot)$ is the imaginary part and $\operatorname{Tr}(\cdot)$ is trace operator. It is straightforward to verify the total degrees of freedom are respected through $\int_{0}^{\infty} \Omega(\omega) d \omega=3 N$, whilst the classical vibrational entropy of the system at a given temperature $T$ :

$$
S=-k_{B} \int_{0}^{\infty}\left[\ln \left(\frac{\hbar \omega}{k_{B} T}\right)-1\right] \Omega(\omega) d \omega .
$$

Whilst equations Eq.(6) and Eq.(7) provide a clear connection between the phonon Green's function and the vibrational entropy, the normal modes $|\nu\rangle$ are typically delocalized across many atoms, complicating an analysis based on a mapping to localized atomic descriptors. As a result, we now emphasis this same formalism using a local basis set to give the local density of states [53].

\section{B. Local basis for densities of states of phonons}

The local density of states can be deduced directly in the Green's function formalism [53]. Our goal is to replace the delocalized basis $|\nu\rangle$ in the above results with a localized basis $|i \alpha\rangle$, where each basis vector is localized on a coordinate $\alpha$ of an atom $i$. As both bases $|\nu\rangle$ and $|i \alpha\rangle$ are complete, they are related by a rotation in $\mathbb{R}^{3 N}$ :

$$
|\nu\rangle=\sum_{i} \sum_{\alpha} \xi^{i \alpha}(\nu)|i \alpha\rangle,
$$

the square of the rotation matrix elements, the $\left|\xi^{i \alpha}(\nu)\right|^{2}$, can be seen as the probability of the phonon $|\nu\rangle$ to be localised on the atom $i$ and along the $\alpha$ direction. By direct substitution into Eq.(5) and using generic properties of rotation matrices we obtain

$$
\begin{aligned}
& \varrho^{i \alpha}(\omega)=\frac{2 \omega}{\pi} \Im\left(\mathcal{G}_{i \alpha}(\omega)\right) . \\
& \varrho^{i \alpha}(\omega)=\sum_{\nu}\left|\xi^{i \alpha}(\nu)\right|^{2} \delta\left(\omega-\omega_{\nu}\right),
\end{aligned}
$$

where $\varrho^{i \alpha}$ is the local DOS of phonon projected on the atom $i$ following the $\alpha$ direction. The classical vibrational entropy of the system from Eq.(7), can then be written as local entropy contribution of each atom:

$$
\begin{aligned}
S & =\sum_{i}[\underbrace{\left.-k_{B} \sum_{\alpha} \sum_{\nu}\left[\ln \left(\frac{\hbar \omega_{\nu}}{k_{B} T}\right)-1\right]\left|\xi^{i \alpha}(\nu)\right|^{2}\right]}_{S_{i}, \text { local information }} \\
& =\sum_{i}\left[\sum_{\alpha} s^{i \alpha}\right]
\end{aligned}
$$

where $s^{i \alpha}$ accounts for the local entropy from the $i^{\text {th }}$ atom in the $\alpha$ direction and $S_{i}=\sum_{\alpha} s^{i \alpha}$ represents the total contribution from the same atom. The above equation is a consequence also of the fact that total density of states of phonons is the sum of the local contribution:

$$
\Omega(\omega)=\sum_{i}\left[\sum_{\alpha} \varrho^{i \alpha}(\omega)\right] .
$$

The redistribution of the total entropy of the system into local contribution, Eq.(11), is exact, as is the total density of states, Eq.(12). As with the local density of states, the local entropy is related to the local environment of the atom. It should be noted that local entropies gather the full spectrum of the dynamical matrix. Green formalism allows to formulate the vibrational entropy problem as a linear problem of sources in term of local geometric environments. This formalism describes long range interactions in term of source as linear elasticity describes long range interactions in term of elastic dipoles.

\section{MACHINE-LEARNING SURROGATE MODEL FOR THE VIBRATIONAL ENTROPY}

Prediction of the vibrational entropy $S$ directly from the relaxed atomic coordinates is impractical due to the high dimension of the input space and the highly nonlinear regression required. In addition, physical constraints such as extensivity in $N$ and $V$ or symmetry 
under exchange of identical atoms are very hard to enforce.

In common with the majority of machine learning models [29, 34, 39, 41, 43], we instead replace a highly complex nonlinear regression task on atomic coordinates $\boldsymbol{q} \in \mathbb{R}^{3 N}$ with a much simpler linear regression task on nonlinear descriptor functions of the atomic coordinates, which we dubbed linear-descriptor machine learning (LDML) model. Often, the effective dimension of the descriptor space is larger than the dimension of the original input space [35-37, 43, 56], though in the present work our mapping will achieve a significant dimensional reduction. The precise choice of descriptor functions is presented in section III A.

To build LDML model, we first assume that the most general model input is a set of $N$ evaluations of $\mathcal{D}$ descriptor functions, giving a descriptor vector $\underline{D}^{i} \in \mathbb{R}^{\mathcal{D}}$ for each atom $i$. To build $\underline{D}^{i}$, the $\mathcal{D}$ descriptor functions take as input the atomic environment around an atom $i$. The atomic environment around $i$ can in principle be the entire system, a point we return to in the next section. This procedure thus initially maps an input space of $\mathbb{R}^{3 N}$ to a descriptor space of $\mathbb{R}^{\mathcal{D} \times N}$. We then assume that these descriptor functions are sufficiently diverse and well chosen such that the local entropy for an atom $i$ can be written as the linear relation

$$
\left[\sum_{\alpha} s^{i \alpha}\right]=\underline{w}^{i} \cdot \underline{D}^{i}
$$

where $\underline{w}^{i} \in \mathbb{R}^{\mathcal{D}}$ is a vector of $\mathcal{D}$ weights. In principle, the total entropy $S=\sum_{i=1}^{N}\left[\sum_{\alpha} s^{i \alpha}\right]$ then requires the determination of $\mathcal{D} N$ parameters for the $\left\{\underline{w}^{i}\right\}$; however, whilst linearity in the $\underline{w}^{i}$ is sufficient to give a thermodynamically extensive entropy [57], to respect symmetry under identical exchange we further require that the weight vectors are identical amongst indistinguishable atoms, implying that $\underline{w}^{i}=\underline{w}$ for the elemental systems considered here, i.e. all weight vectors are identical. This gives a total entropy of

$$
S=\sum_{i=1}^{N}\left[\sum_{\alpha} s^{i \alpha}\right]=\underline{w} \cdot\left(\sum_{i=1}^{N} \underline{D}^{i}\right)=N \underline{w} \cdot\langle\underline{D}\rangle,
$$

where $\langle\underline{D}\rangle=N^{-1} \sum_{i=1}^{N} \underline{D}^{i} \in \mathbb{R}^{\mathcal{D}}$ is the average descriptor vector, meaning that we map the original input $\boldsymbol{q} \in \mathbb{R}^{3 N}$ to a descriptor space of dimension $\mathcal{D} \ll 3 N$ which is system size independent. The predicted entropy Eq.(14) is invariant under identical exchange. Furthermore if one considers $n$ copies of the system then the average vector $\langle\underline{D}\rangle$ over the $\mathrm{n}$ copies is unchanged compared to the original system, thence proving thermodynamic extensivity. The fixed dimensionality of vector $\langle\underline{D}\rangle$ allows the dimension of the input space (i.e. number of atoms) to vary, essential to compute the LDML model formation entropy. Using Eq.(3) we find the formation entropy for a defective system containing $\pm N_{d}$ defects in a bulk lattice of $N_{b}$ atoms :

$$
S_{f}=\left(N_{b} \pm N_{d}\right) \underline{w} \cdot\left[\langle\underline{D}\rangle_{d}-\langle\underline{D}\rangle_{b}\right],
$$

where $\langle\underline{D}\rangle_{d}$ and $\langle\underline{D}\rangle_{b}$ are the average descriptor vectors for the defect-containing and bulk systems, respectively. The formation entropy is therefore the inner product between the model weight vector $\underline{w}$ and the difference in the average descriptor vectors $\langle\underline{D}\rangle_{d}-\langle\underline{D}\rangle_{b}$, multiplied by the total number of atoms. Eq.(15) is a central result of this paper, defining our LDML model. Whilst many choices for machine learning based surrogate models exist, including the popular kernel methods and neural networks [30, 56], the conceptually simpler approach followed here offer many advantages in transferability, overfitting control and analytic connection to thermodynamic properties. In the next section we consider candidate descriptor functions.

\section{A. Choice of descriptor functions}

We have seen that the input vector to our LDML model is the total descriptor vector $N\langle\underline{D}\rangle$, which is symmetric under identical exchange. However, the choice of descriptor functions must also preserve the symmetries and the invariances of the local atomic environment. The notion of descriptor in material science was introduced by Behler and Parrinello [29-31]. They proposed the $\mathbf{G}_{2}$ descriptor, defined below, that underlines the radial distribution of neighbouring atoms weighted by a Gaussian. Since this pioneering work, many descriptors have been developed by (i) introducing the explicit angular description, as the $\mathbf{G}_{3}$ [29], (ii) using the spectral decomposition in $3 D$ or $4 D$ spherical functions of the atomic density [32, 33] (iii) particular design for a given system [45, 58-61] (iv) using even machine / deep learning methods in order to find the appropriate descriptors [62-65] (v) hybrid descriptors that can mix all others classes mentioned above [34]. We note that there are also particular type of descriptors that take the fingerprint of the whole system, offering significant advantages when the observable targeted by the surrogate model cannot be described as a sum of local quantities. Within this particular formalism, the full atomic density is decomposed through a multi-scale convoluted wavelet network, giving a vector of atomdelocalized scattering coefficients [35-38]. This method is particularly relevant for coarse-graining systems where several scales interact. The dimension of descriptors is flexible and is often used to control the level of the accuracy necessary to represent the local atomic environment in the descriptor space. There is therefore always a trade off between computational efficiency, accuracy, and the sensitivity to overfitting which can arise for large input space dimensions. This work compares three local descriptors: the Angular Fourier Series (AFS) [33], the bispectrum $\mathrm{SO}(4)(\mathrm{bSO}(4))[32,33]$ and a scattering 
transform descriptor [35, 36].

The AFS descriptor $\mathcal{A}_{n, l}$ combines the radial and angular information of the local atomic environment. The $n$ and $l$ components account for the radial and angular information of the neighbourhood structure centered on the $i$ atom; defining as $\mathcal{R}_{i}$ the set of indices for atoms less than $r_{c u t}$ from $i$, we have

$$
\mathcal{A}_{n, l}^{i}=\sum_{k, k^{\prime} \in \mathcal{R}_{i}} g_{n}\left(r_{i k}\right) g_{n}\left(r_{i k^{\prime}}\right) \cos \left(l \theta_{i k, i k^{\prime}}\right) f_{i}\left(r_{i k}\right) f_{i}\left(r_{i k^{\prime}}\right),
$$

where $r_{i k}$ is the distance between atom $i$ and atom $k$ and $\theta_{i k, i k^{\prime}}$ the angle formed by the triplet of atom $i, k, k^{\prime}$ centred on $i$. The sum involves the pairs and the triplets of atoms formed by the central $i^{\text {th }}$ atom and the neighbouring atoms inside the sphere with the radius $r_{c u t}$ around atom $i . f$ is a cut-off function, which for the distances $r \geq r_{\text {cut }}$ gives $f_{i}(r) \equiv 0$. The radial functions $g_{n}$ are decreasing polynomials with the distance $r$ having the degree of $\alpha+2$ for $0 \leq \alpha \leq n$. The angular functions are the Tchebyshev polynomials [33] with $0 \leq l \leq l_{\max }$. As $\mathcal{A}_{n, l}$ is formed from a product of the radial and angular functions, the descriptor has a total of $n_{\max }\left(l_{\max }+1\right)$ components. The AFS descriptor enables wide-ranging level of accuracy on radial and angular information by imposing $n_{\max }$ and $l_{\max }$, respectively. Otherwise stated, in this paper we have used $n_{\max }=20$, and $l_{\max }=10$ and the cut-off distance of $5 \AA$. The total number of components for the AFS descriptor used here therefore is 220 .

The bSO(4) descriptor $b S O(4)_{j_{\max }}$ is a spectral descriptor based on the decomposition of the atomic density in $4 D$ hyper-spherical harmonics [32, 33]. The three components of the vector $\mathbf{r} \in \mathbb{R}^{3}$ can be recast into the three angles of the unit sphere $\mathcal{S}^{4} \in \mathbb{R}^{4}$. The local environment of the $i^{\text {th }}$ atom is described as a density $\rho_{i}(\boldsymbol{r})$, and can be decomposed on the $4 D$ hyper-spherical harmonics basis:

$$
\begin{aligned}
\rho_{i}(\boldsymbol{r}) & =\sum_{k \in \mathcal{R}_{i}} w_{k} \delta\left(\boldsymbol{r}-\boldsymbol{r}_{\boldsymbol{k}}\right), \\
& =\sum_{k \in \mathcal{R}_{i}} \sum_{j=0}^{\infty} \sum_{m, m^{\prime}=-j}^{j} \boldsymbol{c}_{i, j}^{m, m^{\prime}} U_{j}^{m, m^{\prime}}
\end{aligned}
$$

where $w_{k}$ is the species-dependent weight, $\boldsymbol{c}_{i, j}^{m, m^{\prime}}$ are the result of the scalar product between the density centred on atom $i$ and the hyper-spherical harmonic $U_{j}^{m, m^{\prime}}$. From the above equation, and the $\boldsymbol{c}_{i, j}^{m, m^{\prime}}$ coefficients, it can be deduced the power and the bi-spectrum of the atomic density. The bi-spectral components of bSO(4) are defined by the following equation, where $j \leq j_{\max }$ and $\left|j_{1}-j_{2}\right| \leq j \leq j_{1}+j_{2}$ :

$$
B_{j j_{1} j_{2}}^{i}=\left(\boldsymbol{c}_{i, j}^{m, m^{\prime}}\right)^{\dagger} \boldsymbol{H}^{j_{1} j_{2}}\left(\boldsymbol{c}_{i, j_{1}}^{m_{1}, m_{1}^{\prime}} \otimes \boldsymbol{c}_{i, j_{2}}^{m_{2}, m_{2}^{\prime}}\right),
$$

where $\boldsymbol{H}^{j_{1} j_{2}}$ is related with the Clebsch-Gordan coefficient of $\mathrm{SO}(4)$ group. A detailed description can be found in $[32,33]$. Following the analysis of the results of the first trial regressions, presented in section $\mathrm{V}$, in this study we use the $j_{\max }=3.5$ and select only the diagonal components $j_{1}=j_{2}[32,33,66]$ yielding in the total number of components to 26 , the cut-off distance is set to $5 \AA$.

The solid harmonic wavelet scattering transform [35, 36 is a multi-scale translation-rotation invariant descriptor. First a global density $\rho$ is computed as a sum of Gaussian functions $g$ centered at the atomic positions:

$$
\rho(\boldsymbol{r})=\sum_{i} g\left(\boldsymbol{r}-\boldsymbol{r}_{\boldsymbol{i}}\right)
$$

Scattering coefficients $S^{\mathcal{J}, L} \rho[j, \ell], j \in \mathcal{J}, 0 \leq l \leq L$ are then computed with convolutions of this density $\rho$ with solid harmonic wavelets $\psi_{j, \ell}^{m}$ of scale $j \in \mathcal{J}$, followed by an integral to have the rotation-translation invariance:

$$
\begin{aligned}
S^{\mathcal{J}, L} \rho[j, \ell] & =\int_{\mathbb{R}^{3}}\left(\sum_{m=-\ell}^{\ell}\left|\rho * \psi_{j, \ell}^{m}(\boldsymbol{r})\right|^{2}\right)^{1 / 2} \mathrm{~d} \boldsymbol{r} \\
\psi_{j, \ell}^{m}(\boldsymbol{r}) & =\frac{1}{(\sqrt{2 \pi})^{3}} e^{-\frac{1}{2}\left|\frac{r}{2^{j}}\right|^{2}}\left|\frac{\boldsymbol{r}}{2^{j}}\right|^{\ell} Y_{\ell}^{m}\left(\frac{\boldsymbol{r}}{|\boldsymbol{r}|}\right) .
\end{aligned}
$$

In this paper, we have used $L=9$ and 9 scales $\mathcal{J}=$ $\{0,0.25,0.5,0.75,1,2,3,4,5\}$ yielding a descriptor of dimension 90 .

\section{TRAINING LDML MODEL}

\section{A. Production of the configuration database}

Any surrogate model is clearly heavily reliant on the database used for training. In this work, we used the ART method [67-70], following the methodology of previous studies [2], to generate a large number of configurations for small vacancy and interstitial clusters in bcc Fe. All clusters contained between 1-4 removed or additional atoms, which we label as $V_{n}$ and $I_{n}$ respectively, with $n=1,2,3,4$. Despite their apparent simplicity, the energy landscape of such defect configurations is known to have many thousands of binding configurations $[2,4,11]$. To test the sensitivity of our surrogate model to the underlying energy model, all calculations were performed in duplicate using two interatomic potentials for bcc Fe, the embedded atom model (EAM) potential developed by Ackland et al. [48](AM04) and the modified embedded atom model (MEAM) potential introduced by Alireza and Asadi [49].

After an initial period of structure generation, all configurations were pairwise compared to ensure the final database only contained non-equivalent structures. Two configurations are considered as non-equivalent provided that two conditions are verified : (i) their energies differ by more than $10^{-2} \mathrm{eV}$; (ii) in the case of interstitial defects the sum of squares of the principal components of 
inertia tensor are different. Interstitial atoms are localized using the Wigner-Seitz method [71].

The resulting database is one order of magnitude larger than that obtained in our previous work [2], due to a wider exploration of phase space with ARTn. The resulting database is summarised in Tab.I.

\begin{tabular}{|c|c|c|c|c|c|}
\hline \multirow{2}{*}{ System $(N, \epsilon)$} & \multicolumn{4}{|c|}{ Type of point defects $\left(N_{c f}\right)$} & \multirow{2}{*}{ Total } \\
\hline & $I_{2}$ & $I_{3}$ & $I_{4}$ & $V_{4}$ & \\
\hline $1024, \epsilon=+0 \%$ & 434 & 1105 & 1280 & 1701 & 4520 \\
\hline $1024, \epsilon=-1 \%$ & 434 & 1105 & 1280 & 1701 & 4520 \\
\hline $1024, \epsilon=+1 \%$ & 434 & 1105 & 1280 & 1701 & 4520 \\
\hline $1024, \epsilon=+2 \%$ & 434 & 1105 & 1280 & 1701 & 4520 \\
\hline $1024, \epsilon=+3 \%$ & 434 & 1105 & 1280 & 1701 & 4520 \\
\hline $2000, \epsilon=+0 \%$ & 434 & 1105 & 1280 & 1701 & 4520 \\
\hline $3456, \epsilon=+0 \%$ & 434 & 1105 & 1280 & 1701 & 4520 \\
\hline Total & 3038 & 7735 & 8960 & 11907 & 31640 \\
\hline
\end{tabular}

TABLE I: Database used for training the present regression model. $N$ is the number of atom in the perfect system, $N_{c f}$ the number of distinct instances for a point defect class.

$I_{2-4}$ and $V_{4}$ denotes the interstitial clusters with 2 up to 4 SIAs and the quadri-vacancy, respectively. The sizes of these systems with defects are $N+(2 \ldots 4)$ and $N-4$ for $I_{2-4}$ and $V_{4}$, respectively. $\epsilon$ is the isotropic and homogeneous rate of deformation for the system

The local descriptors of the retained configurations were computed using the MILADY package $[34,72]$ and the scattering coefficients using the PyScatHarm package [36]. To compute the harmonic entropy for each configuration, the Hessian was computed from $3 N$ force evaluations using the standard finite difference formula with a displacement of $10^{-3} \AA$. Each configuration was tested to be a minimum by checking that the eigen-frequencies are real. For each configuration, we perform an energy relaxation by using LAMMPS [73]. Then, the phonon spectrum and vibrational entropy are computed using PHONDY package [74-77].

\section{B. Regression procedure}

We wish to choose a parametrisation $\underline{w}$ for the LDML model Eq.(14) which is able to approximate the $M$ calculated entropies $\underline{S} \in \mathbb{R}^{M}$ from the $M$ total descriptor vectors $\underline{\underline{D}} \in \mathbb{R}^{\overline{\mathcal{D}} \times M}$. The general training procedure takes a random subset of $M_{t}<M$ entropies $\underline{S}_{t} \in \mathbb{R}^{M_{t}}$ and descriptor vectors $\underline{\underline{D}}_{t} \in \mathbb{R}^{\mathcal{D} \times M_{t}}$, performs a multilinear regression to determine $\underline{w}$, then tests the prediction against the remaining $M_{r}=M-M_{t}$ entropies $\underline{S}_{r} \in \mathbb{R}^{M_{r}}$ and descriptor vectors $\underline{\underline{D}}_{r} \in \mathbb{R}^{\mathcal{D} \times M_{r}}$ by taking statistical measures of the vector-valued training error $\underline{S}_{t}-\underline{w} \cdot \underline{\underline{D}}_{t}$ and test error $\underline{S}_{r}-\underline{w} \cdot \underline{\underline{D}}_{r}$. As it is standard in machine learning development, we compare both the root mean square (RMSE) and mean absolute (MAE) errors. By defining the $L_{p}$ magnitude $\|\underline{v}\|^{p}$ of a vector $\underline{v} \in \mathbb{R}^{M}$ as $\|\underline{v}\|^{p} \equiv \sum_{l=1}^{M}\left|v_{l}\right|^{p}$, the RMSE and MAE errors for the vector-valued error $\underline{S}_{s}-\underline{w} \cdot \underline{\underline{D}}_{s} \in \mathbb{R}^{M_{s}}$ read

$$
\begin{array}{cc}
\sqrt{M_{s}^{-1}\left\|\underline{S}_{s}-\underline{w} \cdot \underline{D}_{s}\right\|^{2}} & \text { (RMSE), } \\
M_{s}^{-1}\left\|\underline{S}_{s}-\underline{w} \cdot \underline{\underline{D}}_{s}\right\|^{1} & \text { (MAE) }
\end{array}
$$

where $s=t$ gives the training error and $s=r$ the test error.

Whilst multilinear regression is conceptually simple, in practice the optimal parametrisation can be difficult to obtain when the number of parameters (here the descriptor vector dimension $\mathcal{D}$ ) is large. The purpose of standard regression is to minimize the $L_{2}$ error : $\left\|\underline{S}_{t}-\underline{w} \cdot \underline{\underline{D}}\right\|_{t} \|^{2}$ with respect to $\underline{w}$. However this can lead to overfitting or highly heterogeneous parametrisation. In order to avoid such difficulties we used a ridge regression where a penalty term is added in the minimisation : $\left\|\underline{S}_{t}-\underline{w} \cdot \underline{D}_{t}\right\|^{2}+\lambda\|\underline{w}\|^{2}$. To optimize the parameter $\lambda$ we use Bayesian ridge regression, a probabilistic generalization of multilinear ridge regression that was applied commonly in the machine learning [56].

Briefly, in the Bayesian approach one models the error $\underline{S}_{t}-\underline{w} \cdot \underline{\underline{D}}_{t} \in \mathbb{R}^{M_{t}}$ as a multidimensional Gaussian random variable with a diagonal covariance matrix $\sigma^{2} \mathbb{I}_{M_{t}}$. In the language of Bayesian estimation, $\sigma$ is a hyperparameter of our estimation procedure, to be distinguished from the model parameters $\underline{w}$ which we want to estimate. This gives a Gaussian likelihood of observing output data $\underline{S}_{t}$ given model parameters $\underline{w}$, input data $\underline{\underline{D}}_{t}$ and error variance $\sigma^{2}$ of

$$
L\left(\underline{S}_{t} \mid \underline{w}, \underline{\underline{D}}, \sigma\right) \propto \exp \left(-\left\|\underline{S}_{t}-\underline{w} \cdot \underline{\underline{D}}_{t}\right\|^{2} / 2 \sigma^{2}\right),
$$

which is clearly a Gaussian of the $L_{2}$ loss function $\left\|\underline{S}_{t}-\underline{w} \cdot \underline{D}_{t}\right\|$ with variance $\sigma^{2}$. A prior distribution of the model parameters $\underline{w}$ is required and as it is standard in a Bayesian approach, we chose another multidimensional Gaussian $p_{0}\left(\underline{w} \mid \sigma_{w}\right)=\exp \left(-\|\underline{w}\|^{2} / 2 \sigma_{w}^{2}\right)$ with $\sigma_{w}$ as the second and final hyperparameter. The product of the prior distribution with the likelihood $L\left(\underline{S}_{t} \mid \underline{w}, \underline{D}, \sigma\right) p_{0}\left(\underline{w} \mid \sigma_{w}\right)$ gives a Gaussian of the ridge regularized $\bar{L}_{2}$ loss function with $\lambda=\sigma^{2} / \sigma_{w}^{2}$. The prior distributions $p_{0}(\sigma), p_{0}\left(\sigma_{w}\right)$ for the hyperparameters are chosen as Gamma distributions, which can be shown to facilitate the analytical derivations when using Gaussian likelihoods [56]. In practice, the hyperparameters reflect the confidence in the final parametrisation.

Defining integrals over the joint hyperparameter prior $p_{0}(\sigma) p_{0}\left(\sigma_{w}\right)$ as $\int_{\sigma, \sigma_{w}} \ldots$, the posterior distribution for $\underline{w}$ given training data $\underline{S}_{t}, \underline{\underline{D}}_{t}$ reads

$$
p\left(\underline{w}_{\mid} \mid \underline{S}_{t}, \underline{\underline{D}}_{t}\right)=\mathcal{N} \int_{\sigma, \sigma_{w}} L\left(\underline{S}_{t} \mid \underline{w}, \underline{\underline{D}}_{t}, \sigma\right) p_{0}\left(\underline{w} \mid \sigma_{w}\right)
$$

where $\left.\mathcal{N}^{-1}=\int \mathrm{d}^{\mathcal{D}} \underline{w} \int_{\sigma, \sigma_{w}} L\left(\underline{S}_{t} \mid \underline{w}, \underline{\underline{D}} t, \sigma\right) p_{0}\left(\underline{w} \mid \sigma_{w}\right)\right\rangle_{\sigma, \sigma_{w}}$ ensures normalization. We aim to find the mode 

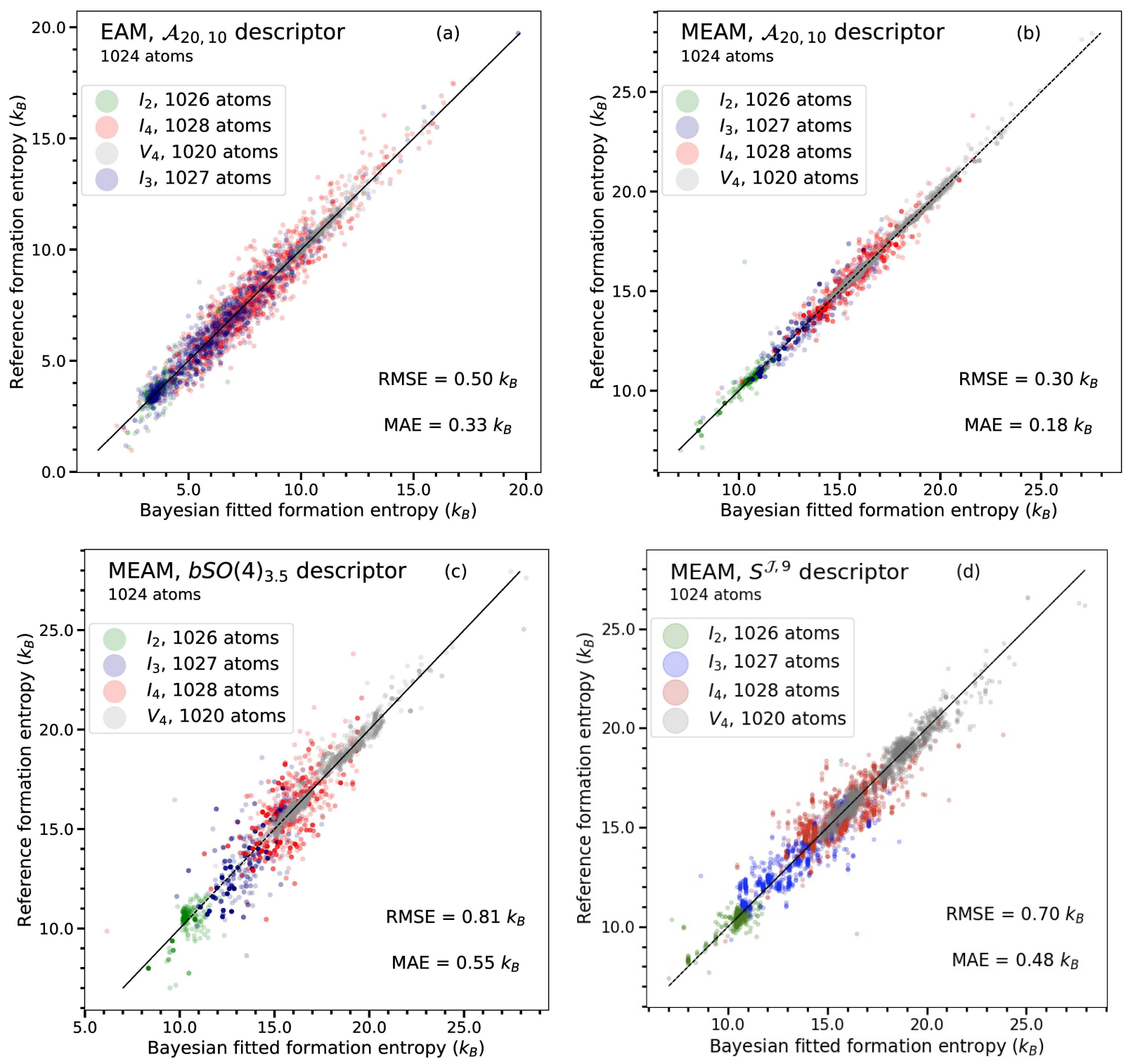

FIG. 2: Formation entropy computed from the numerical diagonalisation of Hessian against the formation entropy computed from LDML model using (a) EAM and (b-d) MEAM dataset for 2-4 interstitial clusters $I_{2-4}$ and for quadri-vacancies $V_{4}$ in $\left(8 a_{0}\right)^{3}$ supercells. The number of configurations are given at first line of Tab.I. The descriptors in the present study are (a-b) $\mathcal{A}_{20,10}$, (c) $b S O(4)_{3.5}$ and (d) $S^{\mathcal{J}, L}$ with scales $\mathcal{J}=\{0,0.25,0.5,0.75,1,2,3,4,5\}$.

of the posterior distribution to determine the optimal parametrisation $\underline{w}$. This is equivalent to maximizing any monotonic function of the posterior with respect to $\underline{w}$, in particular the logarithm, which avoids calculation of the normalization constant $\mathcal{N}$. Our final variational problem for Bayesian ridge regression is thus

$$
\underline{w}=\arg \max _{\underline{w}^{\prime} \in \mathbb{R}^{\mathcal{D}}} \log \int_{\sigma, \sigma_{w}} L\left(\underline{S}_{t} \mid \underline{w}^{\prime}, \underline{\underline{D}}_{t}, \sigma\right) p_{0}\left(\underline{w}^{\prime} \mid \sigma_{w}\right),
$$

which is typically the most stable method to determine the optimal parametrisation. Bayesian linear regressions have been performed by using the scikit-learn package [78]. The initial value of $\sigma_{w}$ for the prior is set by default in the code.

\section{TESTING OF THE LDML MODEL}

\section{A. Influence of interatomic potential and descriptor set}

The LDML model formalism was tested on bcc defect systems as described above (Tab.I), initially in a supercell of size $8 a_{0} \times 8 a_{0} \times 8 a_{0}$. The bulk lattice contained 1024 atoms before the introduction of 2-4 interstitial atoms to produce $I_{2-4}$ defects or removal of 4 atoms for the $V_{4}$ quadri-vacancies. The volume of the defected supercell 

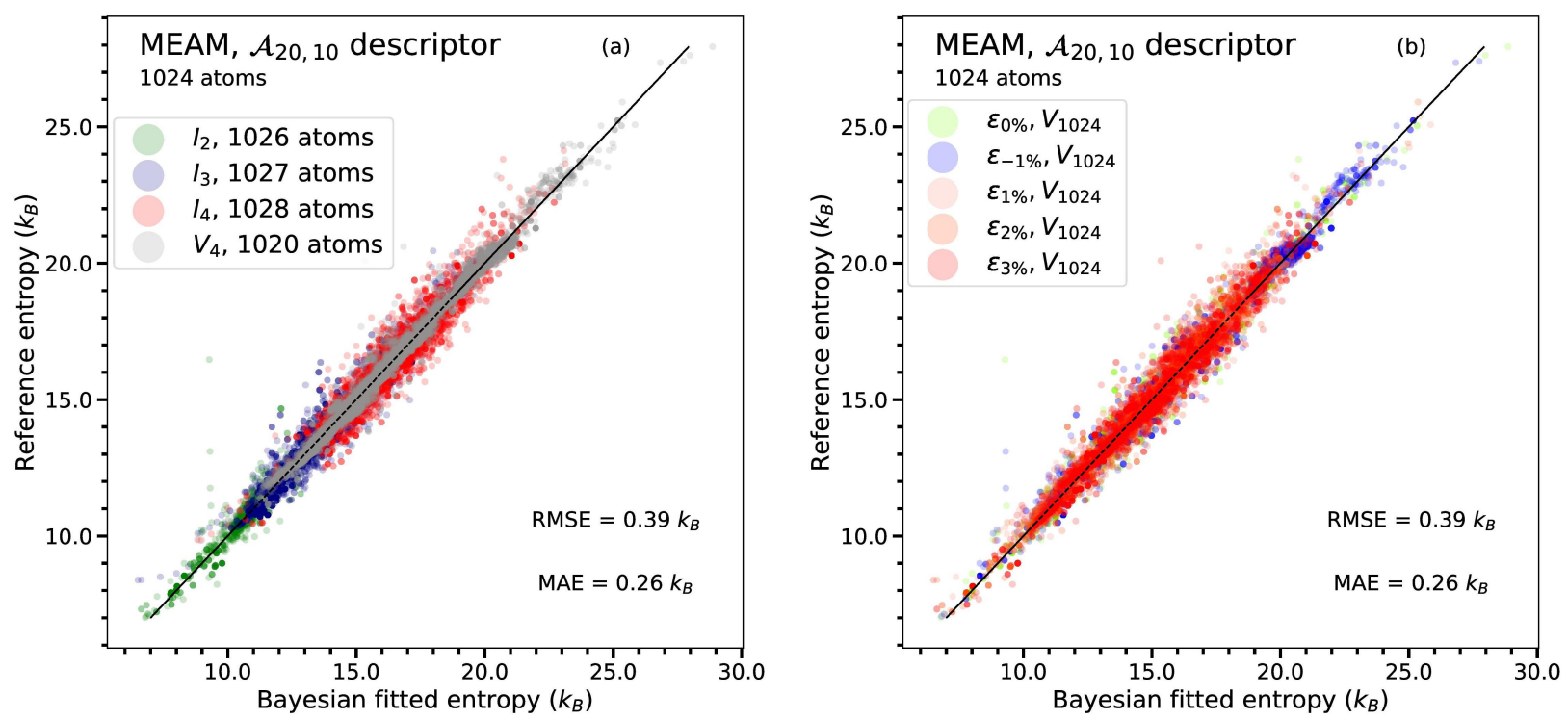

FIG. 3: Illustration of the performance of the training of the surrogate model using deformed supercells of $I_{2-4} / V_{4}$ clusters (MEAM database) with the $\mathcal{A}_{20,10}$ descriptor. The initial configurations have a $\left(8 a_{0}\right)^{3}$ volume and have been deformed by applying an homogeneous and isotropic dilatation of the supercell. The deformation ranges from $-1 \%$ to $3 \%$. (a) The figure illustrates the results of the regression model depending on the type of defect in the supercell; (b) same as in (a) but for the quadri-vacancy $V_{4}$ and various deformation rate.

is fixed to the equilibrium bulk volume.

We first tested the influence of the underlying interatomic potentials by training and testing the LDML model on either EAM [48] or MEAM [49] datasets. The MEAM potential augments the EAM potential form with angular three body terms and typically employs analytic expressions for the pair and embedding functions [79-81] as opposed to the tabulated cubic splines of EAM potentials. We also compared three sets of descriptors for LDML model : (i) $\mathcal{A}_{20,10}$ descriptors, (ii) $b S O(4)_{3.5}$ bispectrum, both with $r_{c u t}=5.0 \AA$, and (iii) the global Scattering descriptor $S^{\mathcal{J}, L}$. In our tests the $\mathcal{A}_{20,10}$ was around $50 \%$ faster to evaluate than $b S O(4)_{3.5}$ and $S^{\mathcal{J}, 9}$

For the three descriptors the MEAM results have a lower RMSE and MAE (see Fig.2), a feature we found replicated across other training sets. The present surrogate model estimates the multi-dimensional curvature of the potential energy surface solely from the geometric structure of the minimum basin. Consequently, it is assumed a smooth energy landscape, i.e. mathematically speaking, the underling potential energy surface is a smooth function with regular derivatives. The EAM potential uses spline functions does not satisfy the assumed regularity, inducing error in the fitting procedure. The MEAM force field is coded on smoother functions resulting in a smaller intrinsic error in the regression model. This inconvenient is not related to the capacity of the force field, EAM or MEAM to describe the physics of phonons in bcc iron. In order to reduce the intrinsic error due to the regularity of the force-field in this paper we use the MEAM potential exclusively. [49].
The results of regressions to the MEAM data with different descriptor functions are shown in Fig.2. On formation entropies ranging between $8 k_{B}$ and $28 k_{B}$, the performance in descriptor sets has limited variation but we find that $\mathcal{A}_{20,10}$ consistently outperforms $b S O(4)_{3.5}$, and $S^{\mathcal{J}, 9}$ despite the greater computational efficiency, with an RMSE of $0.8 k_{B}$ and $0.7 k_{B}$ to $0.3 k_{B}$, respectively.

\section{B. Modelling datasets with multiple defect species and variable supercell volume}

It is highly desirable to have predictability on the changes in formation entropy under deformations of the simulation supercell, as this can be used as a proxy for changes in the formation entropy under varying microstructural environments.

In addition, as LDML model formation entropy Eq.(14) receives an input vector of fixed dimension, independent of system size, it is possible to simultaneously train the model on datasets with variable number of atoms.

As a first application, we trained the LDML model on a large dataset of $I_{2-4}$ and $V_{4}$ configurations, found through the ARTn searches. The simulation cell is the same $8 a_{0}$ cubic supercell as above, where each configuration was additionally copied, subjected to an isotropic dilation of $-1 \%$ to $3 \%$ before a new calculation of descriptor vector and harmonic entropy. The number of configuration in the dataset has been increased by a factor 5. Fig. 3 illustrates the accuracy of LDML model using a single weight vector $\underline{w}$ for the entire dataset. We notice 
that RSME error is only $0.4 k_{B}$.

\section{Training on combined disordered and crystalline datasets}

To test the ability of LDML model and descriptor functions to predict formation entropies beyond crystalline structures, we created an additional database of highly disordered structures from an ART $n$ database of $I_{2-4}$ and $V_{4}$ configurations in cubic supercells of dimension $8 a_{0}$, $10 a_{0}$ and $12 a_{0}$, as described in Tab.I. For each configuration, a large number of individual atoms were subjected to random displacements, which creates many Frenkel pairs even after relaxation. Once the relaxation procedure has been realized we obtained a highly defective structure containing up to 22 vacancies and 26 interstitials. The set of such structures will be referred to as the random database. The distribution of defects in the random database is presented in Fig.4. The difference between the number of interstitials and vacancies is conserved before and after the disordering procedure, giving a strong correlation between the effective vacancy and interstitial count. We also present the distribution of formation entropies Fig. 5(b) and the distribution of distances between point defects Fig. 5(a) associated to Fig. 4. The distribution of formation entropies Fig. 5(b) emphasizes that the selected configurations are diverse and carefully selected. Concerning the distribution of distances between defects Fig. 5(a), we can notice that about $1 / 3$ of distances are less than the 'interaction distance' defines by $2 r_{c u t}=10 \AA$ for the descriptors. Moreover, the interaction between defects is not limited to the cut-off distance of the force field. The point defects used in the present database have strong elastic dipole tensor $[3,82-84]$ that induces a strong elastic field far beyond the defects and made that almost all the defects interact with each other. Fig.6(a) presents the results of LDML model trained on this highly diverse dataset. We find that the RSME error is only $0.8 k_{B}$, which is to be compared to a formation entropy range of approximately 250 $k_{B}$. This high value of formation entropy in comparison to Artn database Fig.2, is ascribed to the much higher effective number of defects in the system.

In order to prove the robustness and the transferability of the model illustrate in the Fig.6(a), a train/test procedure is performed. The database is split randomly into two sets following the proportion $p$. One set corresponds to the training set with the proportion $(1-p)$, the second one is the test set with $p$ proportion. The LDML model is adjusted on the training set and a prediction is realised for the test set. In order to reduce bias on the random procedure we iterate this train/test set sampling hundred times for a given proportion $p$ and we average the values of RMSE and MAE for the training and test set. RMSE and MAE calculated for both sets are presented in inset of Fig.6(a). The weak variability of RMSE against the proportion $p$ indicates the extremely good quality of predictions, up to a splitting of $90 \%$. We notice for $90 \%$ train / $10 \%$ test ratio, that test error is less than train error. This behaviour reflects natural tendency of any regression: more are data in train therefore more the RMSE train is higher. Moreover, at that unbalanced ratio it is possible to have some stochastic fluctuations. So far, the probability to have predicted data with a large systematic error (symptomatic data) is higher than the probability to find symptomatic data in the test set.

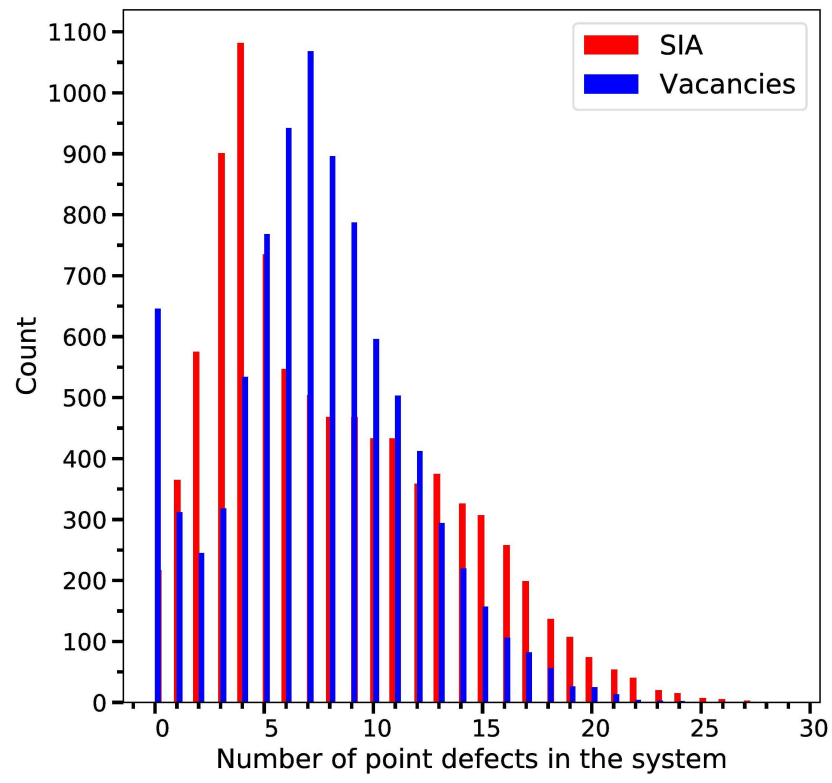

FIG. 4: The analysis of the distribution of randomly generated point defects in the random database. This database is derived from the ARTn database only using the supercells of volume $\left(10 a_{0}\right)^{3}$ and $\left(12 a_{0}\right)^{3}$ by random creation of Frenkel pairs. The plot emphasises the occurrences in the entire random database of number of self interstitial atoms and vacancies in the same supercell. The random database contains 9016 configurations.

Defects from random database are representative structures of bcc iron under irradiation. These structural properties of defects influence drastically the phonon properties. Let's take the case of $\langle 111\rangle$ interstitial clusters $[75,85,86]$. These interstitial clusters exhibit a soft mode due to an almost free translation of the dumbbell along the $\langle 111\rangle$ direction. This phonon mode is highly active in the $\alpha-\gamma$ martensitic transition of $\mathrm{Fe}$ as well as the pair kinks nucleation in the $1 / 2\langle 111\rangle$ dislocation [87] and is delocalized over distances larger than $10 \AA$.

The ability of the present LDML model to mimic the physics of those soft modes is nontrivial, as the characteristic wavelength is far beyond the cutoff radius of the descriptors used to sample the local atomic environment. Despite this, the linear regression in the descriptor space 

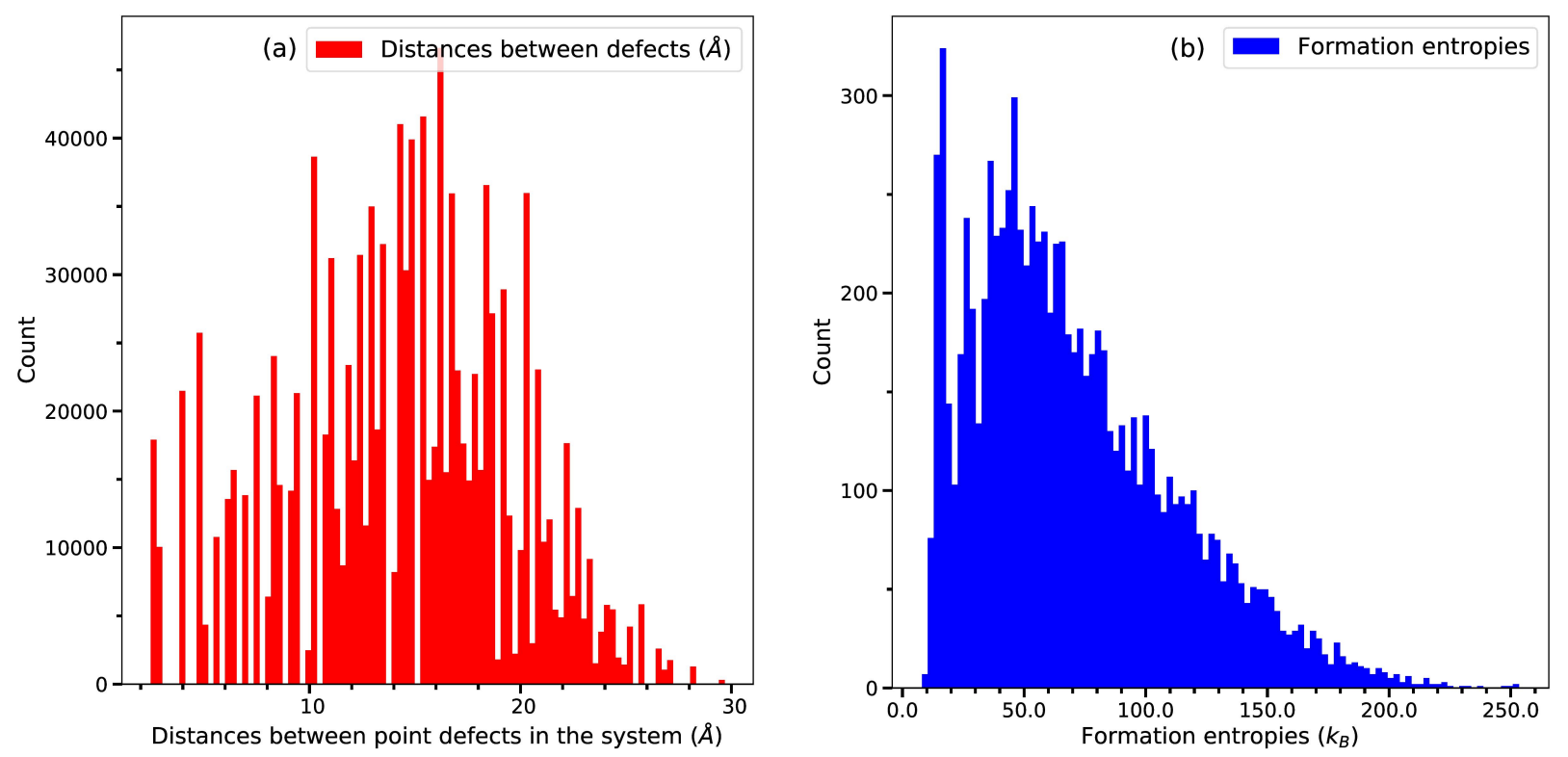

FIG. 5: The analysis of the distribution of formation entropies (b) and of distances between point defects in the random database. Formation entropies follow the same distribution that defect numbers in simulation boxes drawn in Fig. 4. Concerning distances distribution, about $1 / 3$ of point defects are separated by less than $10 \AA=2 r_{\text {cut }}$.

is able to reconstruct the correlation between high formation entropies and the large phonons wavelengths.

\section{Transferability of crystalline model to disordered structures}

In this final example, we artificially tested the transferability of LDML model by training only on the ART $n$ database of defect structures, before attempting to predict the formation entropies of the random database. As illustrated in Fig.6(b), the LDML model achieves a remarkable predictive accuracy with an RSME error of only $1.53 k_{B}$. Such a performance is obtained whilst the prediction is made for a bassin of the energy landscape which is disjointed from the training bassin where formation entropy is bounded by $25 k_{B}$.

In order to prove the transferability of the LDML in non-crystalline structures we investigate $L J_{38}$, the Lennard-Jones cluster containing 38 atoms. It is a archetypal system with thousands on minima organised in many attraction basins [88]. This system is often the benchmark for advanced numerical methods in the exploration of the complex energetic landscapes [8991]. We used the $L J_{38}$ database from Cambridge University: http://www-wales.ch.cam.ac.uk/CCD.html, gratefully provided by Prof. David J. Wales. For such a system the cluster entropies could be easily calculated by direct diagonalisation of the Hessian of the system. We randomly chosen up to 10000 different $L J_{38}$ configurations. The present surrogate model used $A F S(20,10)$ descriptor having $r_{c u t}=5 \AA$. The Fig. 7 illustrates the results of regression model for $L J_{38}$, and inset shows the re- sults of the training/test procedure. The surrogate model presents the same score and transferability behaviour as in the case of bcc iron system.

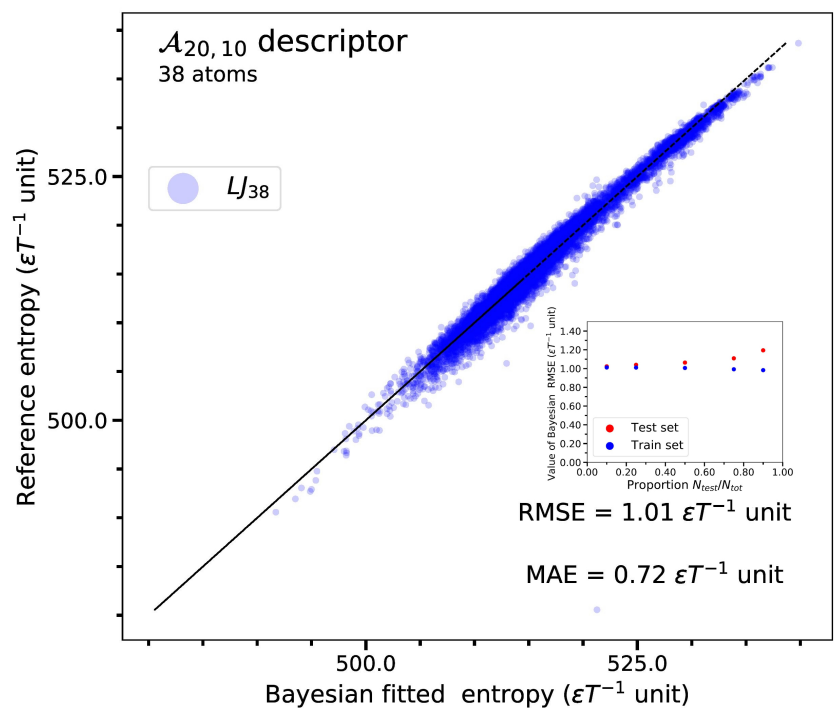

FIG. 7: LDML applied to the Lennard-Jones clusters of 38 atoms $L J_{38}$ to adjust the vibrational entropy of clusters in $\epsilon T^{-1}$ units. Train/test procedure is described Sec. V C results are presented in the inset. The statistical indicators RMSE and MAE remains stable even for large proportions of testing set. 

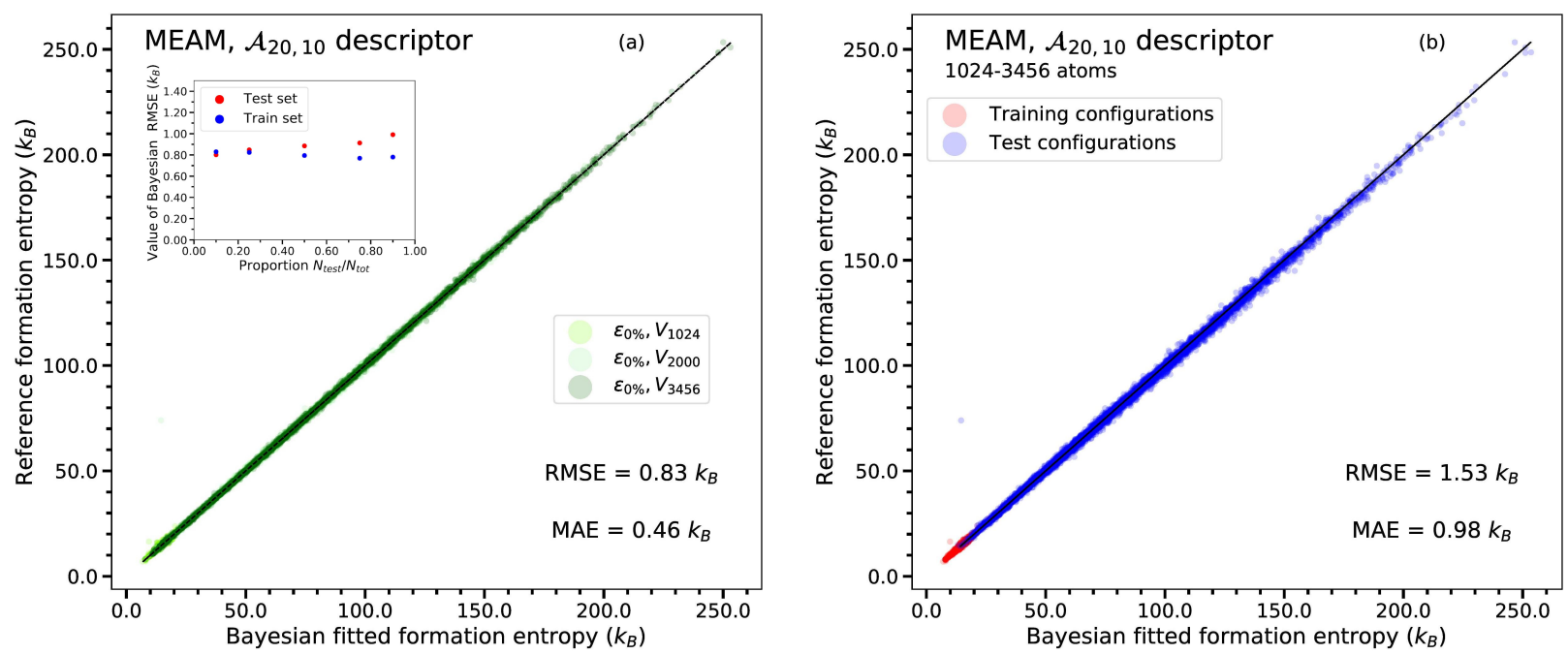

FIG. 6: The robustness and the transferability of the surrogate model is tested by (a) crossing validation using several splitting proportions between train and tested configurations of the joined ARTn database and the random database (the entropies are computed using MEAM potential [49] and the LDML model employs $\mathcal{A}_{20,10}$ descriptor). The statistical indicators are given by the RMSE And MAE. The inset shows RMSE for training and testing dataset against testing proportion (defined in the text). (b) The predictive power of the LDML model trained on the ARTn database and validated on the random database. The statistical indicators, RMSE and MAE, are computed for the random database. The order of magnitude of the statistical indicator are the same as in (a) while the model is in extrapolation regime.

\section{CONCLUSIONS AND PERSPECTIVES}

This work proposes a strategy predict the vibrational entropy of structural defects in crystalline solids from the Cartesian coordinates of atoms. After a training phase, the procedure is based solely on geometrical information and does not require explicit knowledge of the Hessian and its spectrum. The $\mathcal{D}$ chosen descriptor functions are calculated for each atom in a relaxed configuration, then summed across all $N$ atoms, giving a model input space of dimension $\mathcal{D}$ independent of the system size $N$. This reduction is based on the physics of the harmonic approximation that enables to compute the total vibrational entropy of the system as the sum of the local atomic entropies. This reduction is exact within the harmonic approximation and justify the summation over the local descriptors in order to build the descriptor of the simulation box. The regression entropy-descriptor is then parametrised via methods developed in the machine learning community, specifically Bayesian ridge regression. The extensivity for entropy, in number of particules, volume and deformations were carefully checked.

The physics background of the present surrogate model ensures robustness and outstanding transferability. By using two disjoint parts of an extensive database we demonstrate the transferability from supercells containing only one defect cluster to complex configurations having more defects and clusters. The low error in predictions, around $1 \mathrm{k}_{B}$ for the absolute values ranging from $20 \mathrm{k}_{B}$ to $250 \mathrm{k}_{B}$, open many perspectives e.g. the defects can be trained separately in small cells, whilst, compli- cated structures as those in the radiation damage can be accurately predicted [92].

Moreover, the routinely calculations of formation vibrational entropies of defects is hindered by the computational cost. The harmonic approximation scales as cubic in number of atoms. In the present approach, the evaluation is very rapid. The vast majority of the numerical evaluation is reserved by the calculation of the fingerprint of the local atomic environment. The present algorithm scales as linear with the number of atoms and can be easily parallelized for massive systems.

The present surrogate model opens the way for the prediction of the vibrational entropy of defects up to nanometric-size with a host medium up to millions of atoms. Moreover, the present strategy can be integrated in on-the-fly skims such as relaxed Monte Carlo for fast evaluation of the the kinetical pathways of the system on the free energy landscape.

\section{ACKNOWLEDGMENTS}

This work has been carried out within the framework of the EUROfusion Consortium and has received funding from Euratom Research and Training Programme 20192020 under Grant Agreement No. 633053. CL and MCM acknowledge the support from GENCI - (CINES/CCRT) computer centre under Grant No. A0070906973. LT and SM acknowledge the support from ANR-19-P3IA-0001 program of the PRAIRIE 3IA Institute. 
[1] V. V. Bulatov, L. L. Hsiung, M. Tang, A. Arsenlis, M. C. Bartelt, W. Cai, J. N. Florando, M. Hiratani, M. Rhee, and G. Hommes, Nature 440, 1174 (2006).

[2] M.-C. Marinica, F. Willaime, and N. Mousseau, Physical Review B 83, 094119 (2011).

[3] R. Alexander, M.-C. Marinica, L. Proville, F. Willaime, K. Arakawa, M. Gilbert, and S. Dudarev, Physical Review B 94, 024103 (2016).

[4] M.-C. Marinica, F. Willaime, and J.-P. Crocombette, Physical Review Letters 108, 025501 (2012).

[5] C. Woodward and S. I. Rao, Physical review letters 88, 216402 (2002).

[6] D. A. Terentyev, T. P. C. Klaver, P. Olsson, M.-C. Marinica, F. Willaime, C. Domain, and L. Malerba, Physical Review Letters 100, 145503 (2008).

[7] D. Mordehai, E. Clouet, M. Fivel, and M. Verdier, Philosophical Magazine 88, 899 (2008).

[8] O. Y. N., D. J. Bacon, Z. Rong, and B. N. Singh, Philosophical Magazine 84, 745 (2004).

[9] D. J. Wales, Energy Landscapes, edited by C. U. Press (Cambridge, 2003).

[10] D. J. Wales, Journal of Chemical Physics 130, 204111 (2009).

[11] T. D. Swinburne and D. Perez, Physical Review Materials 2, 053802 (2018).

[12] K. Arakawa, M.-C. Marinica, S. Fitzgerald, L. Proville, D. Nguyen-Manh, S. L. Dudarev, P.-W. Ma, T. D. Swinburne, A. M. Goryaeva, T. Yamada, T. Amino, S. Arai, Y. Yamamoto, K. Higuchi, N. Tanaka, H. Yasuda, T. Yasuda, and H. Mori, Nature Materials 19, 508 (2020).

[13] S. S. Kapur, M. Prasad, J. C. Crocker, and T. Sinno, Phys. Rev. B 72, 014119 (2005).

[14] A. Satta, F. Willaime, and S. de Gironcoli, Phys. Rev. B 57, 11184 (1998).

[15] G. H. Vineyard and G. J. Dienes, Phys. Rev. 93, 265 (1954).

[16] H. B. Huntington, G. A. Shirn, and E. S. Wajda, Phys. Rev. 99, 1085 (1955).

[17] N. W. Ashcroft and N. D. Mermin, Solid state physics, Holt-Saunders International Editions: Science : Physics (Holt, Rinehart and Winston, 1976).

[18] T. Lelièvre, G. Stoltz, and M. Rousset, Free energy computations: A mathematical perspective (Imperial College Press, London, 2010).

[19] G. M. Torrie and J. P. Valleau, Journal of Computational Physics 23, 187 (1977).

[20] A. Laio and M. Parrinello, Proceedings of the National Academy of Sciences 99, 12562 (2002).

[21] L. Maragliano and E. Vanden-Eijnden, Chemical Physics letters 446, 182 (2007).

[22] E. Weinan, W. Ren, and E. Vanden-Eijnden, Physical Review B 66, 052301 (2002).

[23] T. Lelièvre, M. Rousset, and G. Stoltz, Journal of Chemical Physics 126, 134111 (2007).

[24] L. Bonati and M. Parrinello, Physical Review Letters 121, 265701 (2018).

[25] E. Darve, D. Rodríguez-Gómez, and A. Pohorille, Journal of Chemical physics 128, 144120 (2008).

[26] T. D. Swinburne and M.-C. Marinica, Physical Review Letters 120, 135503 (2018).
[27] L. Mauger, M. S. Lucas, J. A. Muñoz, S. J. Tracy, M. Kresch, Y. Xiao, P. Chow, and B. Fultz, Phys. Rev. B 90, 064303 (2014).

[28] D. Bansal, A. Aref, G. Dargush, and O. Delaire, Journal of Physics: Condensed Matter 28, 385201 (2016).

[29] J. Behler and M. Parrinello, Physical Review Letters 98, 146401 (2007).

[30] J. Behler, Journal of Chemical Physics 134, 074106 (2011).

[31] G. Imbalzano, A. Anelli, D. Giofré, S. Klees, J. Behler, and M. Ceriotti, Journal of Chemical Physics 148, 241730 (2018).

[32] A. P. Bartók, Gaussian Approximation Potential : an interatomic potential derived from first principles Quantum Mechanics, Ph.D. thesis, University of Cambridge, Cambridge (2009).

[33] A. P. Bartók, R. Kondor, and G. Csányi, Physical Review B 87, 184115 (2013).

[34] A. M. Goryaeva, J.-B. Maillet, and M.-C. Marinica, Computational Materials Science 166, 200 (2019).

[35] M. Eickenberg, G. Exarchakis, M. Hirn, and S. Mallat, in Advances in Neural Information Processing Systems 30, edited by I. Guyon, U. V. Luxburg, S. Bengio, H. Wallach, R. Fergus, S. Vishwanathan, and R. Garnett (Curran Associates, Inc., 2017) p. 6540-6549.

[36] M. Eickenberg, G. Exarchakis, M. Hirn, S. Mallat, and L. Thiry, Journal of Chemical Physics 148, 241732 (2018).

[37] M. Hirn, S. Georges Mallat, and N. Poilvert, Multiscale Modeling Simulation 15 (2016).

[38] E. Homer, D. M. Hensley, C. Rosenbrock, A. Nguyen, and G. Hart, Frontiers in Materials 6 (2019).

[39] D. Dragoni, T. D. Daff, G. Csányi, and N. Marzari, Physical Review Materials 2, 013808 (2018).

[40] W. J. Szlachta, A. P. Bartók, and G. Csányi, Physical Review B 90, 104108 (2014).

[41] A. P. Bartók, M. C. Payne, R. Kondor, and G. Csányi, Physical Review Letters 104, 136403 (2010).

[42] C. Chen, Z. Deng, R. Tran, H. Tang, I.-H. Chu, and S. P. Ong, Physical Review Materials 1, 043603 (2017).

[43] A. Thompson, L. Swiler, C. Trott, S. Foiles, and G. Tucker, Journal of Computational Physics 285, 316 (2015).

[44] V. Botu and R. Ramprasad, arXiv:1410.3353 [cond-mat] (2014), arXiv: 1410.3353.

[45] J. R. Kermode, A. Gleizer, G. Kovel, L. Pastewka, G. Csányi, D. Sherman, and A. De Vita, Physical Review Letters 115, 135501 (2015).

[46] L. M. Ghiringhelli, J. Vybiral, S. V. Levchenko, C. Draxl, and M. Scheffler, Physical Review letters 114, 105503 (2015).

[47] L. Dezerald, L. Proville, L. Ventelon, F. Willaime, and D. Rodney, Physical Review B 91, 094105 (2015).

[48] G. J. Ackland, M. I. Mendelev, D. J. Srolovitz, S. Han, and A. V. Barashev, Journal of Physics: Condensed Matter 16, S2629 (2004).

[49] S. A. Etesami and E. Asadi, Journal of Physics and Chemistry of Solids 112, 61-72 (2018).

[50] B. Grabowski, L. Ismer, T. Hickel, and J. Neugebauer, Physical Review B 79, 134106 (2009). 
[51] B. Grabowski, T. Hickel, and J. Neugebauer, Physica Status Solidi (b) 248, 1295 (2011).

[52] A. Glensk, B. Grabowski, T. Hickel, and J. Neugebauer, Physical Review Letters 114, 195901 (2015).

[53] K. S. P. H. Dederichs, R. Zeller, Point Defects in Metals II, Dynamical Properties and Diffusion Controlled Reactions (Springer Tracts in Modern Physics, Berlin, 1980).

[54] Progress in Materials Science 55, 247-352 (2010).

[55] C. Huang, A. F. Voter, and D. Perez, Physical Review B 87, 214106 (2013).

[56] C. E. Rasmussen, Gaussian Processes in Machine Learning (Springer, Berlin, Heidelberg, 2004).

[57] D. Frenkel and S. Berend, Understanding Molecular Simulation: From Algorithms to Applications (Academic Press, 2002).

[58] H. Zong, G. Pilania, X. Ding, G. J. Ackland, and T. Lookman, npj Computational Materials 4, 1 (2018).

[59] G. Ferré, J.-B. Maillet, and G. Stoltz, Journal of Chemical Physics 143, 104114 (2015).

[60] V. Botu, R. Batra, J. Chapman, and R. Ramprasad, Journal of Physical Chemistry C 121, 511 (2017).

[61] E. Cubuk, S. Schoenholz, J. Rieser, B. Malone, J. Rottler, D. Durian, E. Kaxiras, and A. Liu, Physical Review Letters 114, 108001 (2015).

[62] F. Noe and C. Clementi, Journal of Chemical Theory and Computation 11, 5002 (2015).

[63] K. T. Schutt, H. E. Sauceda, P.-J. Kindermans, A. Tkatchenko, and K.-R. Müller, Journal of Chemical Physics 148, 241722 (2018).

[64] A. L. Ferguson, A. Z. Panagiotopoulos, I. G. Kevrekidis, and P. G. Debenedetti, Chemical Physics Letters 509, 1 (2011).

[65] W. F. Reinhart, A. W. Long, M. P. Howard, A. L. Ferguson, and A. Z. Panagiotopoulos, Soft Matter 13, 4733 (2017).

[66] R. Kakarala, The bispectrum as a source of phasesensitive invariants for Fourier descriptors: a grouptheoretic approach, Ph.D. thesis, Irvine University, Irvine (1992).

[67] G. T. Barkema and N. Mousseau, Physical Review Letters $\mathbf{7 7}, 4358$ (1996).

[68] R. Malek and N. Mousseau, Physical Review E - Statistical Physics, Plasmas, Fluids, and Related Interdisciplinary Topics 62, 7723-7728 (2000).

[69] E. Cancès, F. Legoll, M.-C. Marinica, K. Minoukadeh, and F. Willaime, Journal of Chemical Physics 130, 114711 (2009).

[70] E. Machado-Charry, L. K. Béland, D. Caliste, L. Genovese, T. Deutsch, N. Mousseau, and P. Pochet, Journal of Chemical Physics 135, 034102 (2011).

[71] E. Wigner and F. Seitz, Phys. Rev. 43, 804 (1933).

[72] M. C. Marinica and et al, MiLaDy - Machine Lerning Dynamics (CEA, Saclay, 2015-2019).

[73] S. Plimpton, Journal Computational Physics 117, 1 (1995).

[74] M. C. Marinica and et al, Phondy - Phonons Dynamics (CEA, Saclay, 2007-2019).

[75] M.-C. Marinica and F. Willaime, Solid State Phenomena 129, 67 (2007).

[76] A. Soulié, F. Bruneval, M.-C. Marinica, S. Murphy, and J.-P. Crocombette, Physical Review Materials 2, 083607 (2018).

[77] F. Berthier, J. Creuze, T. Gabard, B. Legrand, M.-C. Marinica, and C. Mottet, Physical Review B 99, 014108
(2019).

[78] F. Pedregosa, G. Varoquaux, A. Gramfort, V. Michel, B. Thirion, O. Grisel, M. Blondel, P. Prettenhofer, R. Weiss, V. Dubourg, J. Vanderplas, A. Passos, D. Cournapeau, M. Brucher, M. Perrot, and E. Duchesnay, Journal of Machine Learning Research 12, 2825 (2011).

[79] M. S. Daw and M. I. Baskes, Physical Review B 29, 6443 (1984).

[80] M. S. Daw, S. M. Foiles, and M. I. Baskes, Materials Science Reports 9, 251 (1993).

[81] M. I. Baskes, Physical Review B 46, 2727 (1992).

[82] C. Varvenne, F. Bruneval, M. C. Marinica, and E. Clouet, Physical Review B 88, 134102 (2013).

[83] M. Boleininger and S. L. Dudarev, Phys. Rev. Materials 3, 093801 (2019).

[84] D. R. Mason, D. Nguyen-Manh, M.-C. Marinica, R. Alexander, A. E. Sand, and S. L. Dudarev, Journal of Applied Physics 126, 075112 (2019).

[85] G. Lucas and R. Schäublin, Nuclear Instruments and Methods in Physics Research Section B: Beam Interactions with Materials and Atoms 267, 3009 (2009).

[86] S. Chiesa, P. M. Derlet, and S. L. Dudarev, Physical Review B 79, 214109 (2009).

[87] L. Proville, D. Rodney, and M.-C. Marinica, Nature Materials 11, 845-849 (2012).

[88] D. Wales, Energy Landscapes: Applications to Clusters, Biomolecules and Glasses, Cambridge Molecular Science (Cambridge University Press, 2004).

[89] D. J. Wales, Molecular physics 100, 3285 (2002).

[90] D. J. Wales, Molecular physics 102, 891 (2004).

[91] D. J. Wales, "Cambridge cluster database," .

[92] S. J. Zinkle and B. N. Singh, Journal of Nuclear Materials 199, 173 (1993). 